Pacific Journal of Mathematics

MAXIMAL MEANS AND TAUBERIAN THEOREMS 


\section{MAXIMAL MEANS AND TAUBERIAN THEOREMS}

\section{A. RUBEL}

Introduction. In this paper, we study two well-known mathematical ideas that have hitherto been regarded as unconnected. It is our purpose to show that they are closely related. The first idea is one developed by Pólya in his theory of maximal density [3]. The second is the idea of repeated differentiation introduced by Littlewood in Tauberian arguments [2], [1].

Our account of the Pólya theory is virtually a direct translation of certain sections of [3]. To apply his methods to bounded Lebesguemeasurable functions in general requires only simple changes. Consequently, we state only the results, leaving the proofs to the reader.

Then the idea of Littlewood is developed in a theory of Littlewood means that compares closely, theorem by theorem, with the theory of Pólya means. Thus, Theorems 1.1 and 2.1, Theorems 1.2 and 2.2, etc., should be compared. The theory of Littlewood means may be regarded as the theory of the $p$-fold application of l'Hospital's rule to a certain class of indeterminate forms. The order $p$ is not restricted to positive integral values; indeed, $p$ may be any real number between -1 and $+\infty$.

But the connection between the two theories goes deeper than mere analogy. Our principle result, Theorem 3.1, asserts that the Polya maximal upper mean, $\mathscr{L}(1)$, is equal to the Littlewood maximal upper mean, $\Lambda(\infty)$, and that the minimal lower means are also equal to each other. An immediate corollary of this theorem is the celebrated Tauberian theorem of Littlewood that a bounded and Lebesgue-measurable function has a Cèsaro average if and only if it has an Abel average.

Finally, in $\S 4$, we give an intrinsic characterization of the mean $\mathscr{L}(1)$ as the infimum of the averages of all Cèsaro-averageable functions $f^{*}$ with $f^{*}(x) \geq f(x)$ for all $x$. This might be compared with the characterization of the outer measure of a set as the infimum of the measures of all measurable sets that cover it.

1. The Pólya means. Let $f(x)$ be a given bounded and Lebesguemeasurable function on $(0, \infty)$. For $0<\xi<1$ define

$$
\begin{aligned}
\mathscr{L}(\xi) & =\limsup _{x \rightarrow \infty} \frac{1}{x-\xi x} \int_{\xi x}^{x} f(t) d t, \\
l(\xi) & =\lim \inf \text { (same) } .
\end{aligned}
$$

The quantities $\mathscr{L}(0)$ and $l(0)$ are the ordinary Cèsaro lim sup and

Received November 27, 1958, and in revised form September 30, 1959. Presented to the American Mathematical Society, October 26, 1957. This research was supported by a postdoctoral fellowship from the National Science Foundation. 
lim inf, respectively, of $f$. We define $\mathscr{L}(1)=\lim _{\xi \rightarrow 1} \mathscr{L}(\xi)$ and $l(1)=$ $\lim _{\xi \rightarrow 1} l(\xi)$, where the existence of the limits is guaranteed by Theorem 1.3. We call $\mathscr{L}(1)$ the "maximal Pólya upper mean" and $l(1)$ the "minimal Pólya lower mean".

THEOREM 1.1. $\mathscr{L}(\xi)$ and $l(\xi)$ are continuous functions of $\xi$ for $0 \leq \xi \leq 1$.

THEOREM 1.2. $\mathscr{L}(\xi) \geq \mathscr{L}\left(\xi^{n}\right)$ and $l(\xi) \leq l\left(\xi^{n}\right)$ for each $\xi, 0 \leq \xi \leq 1$ and $n=1,2,3, \cdots$.

THEOREM 1.3. $\mathscr{L}(1)=\lim _{\xi \rightarrow 1-} \mathscr{L}(\xi)$ and $l(1)=\lim _{\xi \rightarrow 1-} l(\xi)$ exist.

REMARK. It may happen that $\mathscr{L}(\xi)$ and $l(\xi)$ fail to be monotone functions. Nevertheless, we have the next theorem.

TheOREM 1.4. For all $\xi, 0 \leq \xi \leq 1, \mathscr{L}(1) \geq \mathscr{L}(\xi) \geq \mathscr{L}(0)$ and $l(1) \leq l(\xi) \leq l(0)$.

THeOREM 1.5. If there are numbers $\xi_{0}$ and $\xi_{1}$ with $0 \leq \xi_{0} \leq 1$, $0 \leq \xi_{1} \leq 1$ for which $l\left(\xi_{1}\right) \geq \mathscr{L}\left(\xi_{0}\right)$, then there is a constant, L, such that $\mathscr{L}(\xi)=l(\xi)=L$ for all $\xi, 0 \leq \xi \leq 1$.

2. The Littlewood means. Let $f(x)$ be a bounded, Lebesguemeasurable function on $(0, \infty)$. The Abel averaging method studies the behaviour of $F(x) / I(x)$ as $x \rightarrow 0+$, where $F(x)=\int_{0}^{\infty} f(t) e^{-x t} d t$ and $I(x)=$ $\int_{0}^{\infty} e^{-x t} d t=x^{-1}$. We regard $F(x) / I(x)$ as an "indeterminate form $\infty / \infty$ " at $x=0$. The Littlewood means arise by applying l'Hospital's rule $p$ times to the fraction $F(x) / I(x)$. Thus, we study also the ratios $F^{(p)}(x) / I^{(p)}(x)$ where

$$
F^{(p)}(x)=(-)^{p} \int_{0}^{\infty} t^{p} e^{-x t} f(t) d t
$$

and

$$
I^{(p)}(x)=(-)^{p} \int_{0}^{\infty} t^{p} e^{-x t} d t=(-)^{p} x^{-(p+1)} \Gamma(p+1) .
$$

Here, $p$ may be any real number in the range $-1<p<\infty$ and we put $(-)^{p}=e^{i \pi p}$. When $p$ is a positive integer, $F^{(p)}(x)$ is the $p$ th derivative of $F(x)$ and $I^{(p)}(x)$ is the $p$ th derivative of $I(x)$. We define

$$
\begin{aligned}
\Lambda(p) & =\limsup _{x \rightarrow 0+} F^{(p)}(x) / I^{(p)}(x) \\
\lambda(p) & =\liminf _{x \rightarrow 0+} F^{(p)}(x) / I^{(p)}(x) .
\end{aligned}
$$


The quantities $\Lambda(0)$ and $\lambda(0)$ are the ordinary Abel lim sup and Abel lim inf, respectively, of $f$. We define $\Lambda(\infty)=\lim _{p \rightarrow \infty} \Lambda(p), \lambda(\infty)=$ $\lim _{p \rightarrow \infty} \lambda(p), \Lambda(-1)=\lim _{p \rightarrow-1+} \Lambda(p)$, and $\lambda(-1)=\lim _{p \rightarrow-1+} \lambda(p)$, where the existence of the limits is guaranteed by Theorem 2.3. We call $\Lambda(\infty)$ the "maximal Littlewood mean" and $\lambda(\infty)$ the "minimal Littlewood mean."

THEOREM 2.1. $\Lambda(p)$ and $\lambda(p)$ are continuous functions of $p$ for $-1 \leq p \leq \infty$.

Proof. We have

$$
\begin{aligned}
\Lambda(q)- & \Lambda(p)=\limsup _{x \rightarrow 0+} F^{(q)}(x) / I^{(q)}(x)-\limsup _{x \rightarrow 0+} F^{(p)}(x) / I^{(p)}(x) \\
& \leq \limsup _{x \rightarrow 0+}\left\{F^{(q)}(x) / I^{(q)}(x)-F^{(p)}(x) / I^{(p)}(x)\right\} \\
& =\limsup _{x \rightarrow 0+} \int_{0}^{\infty}\left\{u^{q} / \Gamma(q+1)-u^{p} / \Gamma(p+1)\right\} f(u / x) e^{-u} d u,
\end{aligned}
$$

with a similar inequality in the opposite direction. Using now the fact that $|f(u / x)|$ is uniformly bounded, standard techniques of estimation may be applied to the integral in (2.1) to show that $\lim _{q \rightarrow p} \Lambda(q)=\Lambda(p)$. The case $p=-1$ and $p=\infty$ follow by definition.

THEOREM 2.2. $\Lambda(p)$ is a non-decreasing function of $p$ and $\lambda(p)$ is a non-increasing function of $p$.

REMARK. Theorem 2.2 is clearly a stronger kind of statement than Theorem 1.2. A direct analogue of Theorem 1.2 would state merely that $\Lambda(p+1) \geq \Lambda(p)$ and $\lambda(p+1) \leq \lambda(p)$. It is interesting to note that there is a special proof of this direct analogue, based on the fact that $F^{(p+1)}(x)$ and $I^{(p+1)}(x)$ are the respective derivatives of $F^{(p)}(x)$ and $I^{(p)}(x)$. We need only put $(-)^{p} g(x)=F^{(p)}(x)$ and $(-)^{p} h(x)=I^{(p)}(x)$ in the following statement of one form of l'Hospital's rule, after $f(x)$ is suitably normalized.

L'Hospital's rule. If $g(x)$ and $h(x)$ are differentiable functions of $x$ for $x>0$ and $g(0+)=\infty$ and $h(0+)=\infty$ then

$$
\limsup _{x \rightarrow 0+} g(x) / h(x) \leq \limsup _{x \rightarrow 0+} g^{\prime}(x) / h^{\prime}(x) .
$$

Proof of Theorem 2.2. The notation of $*$ for Mellin convolution will simplify our proof considerably. If $A(x), B(x), C(x)$ and $D(x)$ are suitably restricted functions on $0<x<\infty$, we define

$$
(A * B)(x)=\int_{0}^{\infty} A(t) B(x / t) \frac{d t}{t},
$$


and see that

$$
A *(B * C)=(A * B) * C, \text { and } A * B=B * A .
$$

Further, if $C(x) \geq 0$ for all $x$ and $(C * 1)(x) \equiv 1$, where $1(x)=1$ for all $x$, then $\lim \sup _{x \rightarrow \infty}(C * D)(x) \leq \lim \sup _{x \rightarrow \infty} D(x)$.

If we now suppose $p<q$ and let

$$
K_{p}(x)=\frac{x^{p+1} e^{-x}}{\Gamma(p+1)}, \quad K_{q}(x)=\frac{x^{q+1} e^{-x}}{\Gamma(q+1)}, \quad g(x)=f\left(\frac{1}{x}\right),
$$

and

$$
K_{p, q}(x)=\left\{\begin{array}{cc}
\frac{\Gamma(q+1)}{\Gamma(p+1) \Gamma(q-p)} x^{p+1}(1-x)^{q-p-1} & \text { for } x<1 \\
0 & \text { for } x \geq 1
\end{array}\right.
$$

then we have

$$
\begin{aligned}
& \Lambda(p)=\limsup _{x \rightarrow \infty}\left(K_{p} * g\right)(x) \\
& \Lambda(q)=\limsup _{x \rightarrow \infty}\left(K_{q} * g\right)(x) \\
& K_{p}=K_{q} * K_{p, q} \\
& K_{p, q} \geq 0 \\
& \left(K_{p, q} * 1\right)(x) \equiv 1 .
\end{aligned}
$$

In accordance with the preceding remarks, it follows that $\Lambda(p) \leq \Lambda(q)$, and we are done.

THEOREM 2.3. $\Lambda(\infty)=\lim _{p \rightarrow \infty} \Lambda(p), \lambda(\infty)=\lim _{p \rightarrow \infty} \lambda(p), \Lambda(-1)=$ $\lim _{p \rightarrow-1+} \Lambda(p)$, and $\lambda(-1)=\lim _{p \rightarrow-1+} \lambda(p)$ exist.

Proof. This is an obvious consequence of the monotonicity of the bounded functions $\Lambda(p)$ and $\lambda(p)$.

TheOREM 2.4. For all $p,-1 \leq p \leq \infty, \Lambda(\infty) \geq \Lambda(p) \geq \Lambda(-1)$ and $\lambda(\infty) \leq \lambda(p) \leq \lambda(-1)$.

Proof. Same as that of Theorem 2.3.

THEOREM 2.5. If there are numbers $p_{0}$ and $p_{1}$ with $-1<p_{0} \leq \infty$, $-1<p_{1} \leq \infty$ for which $\lambda\left(p_{1}\right) \geq \Lambda\left(p_{0}\right)$, then there is a constant, $L$, such that $\Lambda(p)=\lambda(p)=L$ for all $p,-1 \leq p \leq \infty$.

REMARK. As is shown in the example following the proof of this theorem, the values $p_{0}=-1, p_{1}=-1$ must be excluded from the hypo- 
theses. This is in contrast to Theorem 1.5 where the values $\xi_{0}=0$, $\xi_{1}=0$ are not only included in the hypotheses, but play the most important role in the proof. In the example, $f(x)=\sin \log x, \lambda(-1)=$ $\Lambda(-1)=0$, but $\Lambda(\infty)=1$.

Proof of Theorem 2.5. Suppose $p_{1} \geqq p_{0}$. Then $\lambda\left(p_{0}\right) \geqq \lambda\left(p_{1}\right)$ and hence $\lambda\left(p_{0}\right)=\Lambda\left(p_{0}\right)$. If, on the other hand, $p_{1} \leq p_{0}$, then $\Lambda\left(p_{0}\right) \geq \Lambda\left(p_{1}\right)$ and hence $\lambda\left(p_{1}\right)=\Lambda\left(p_{1}\right)$. It is therefore enough to show that if there exists a $p_{0}>-1$ such that $\lambda\left(p_{0}\right)=\Lambda\left(p_{0}\right)$, then $\lambda(p)=\Lambda(p)=$ constant.

Now the following familiar lemma will be enough to show that if $\lambda\left(p_{0}\right)=\Lambda\left(p_{0}\right)=L$, say, then $\lambda\left(p_{0}+1\right)=\Lambda\left(p_{0}+1\right)=L . \quad$ By repeating this argument, we deduce that $\lambda(\infty)=\Lambda(\infty)=L$. But since, for all $p, \lambda(\infty) \leq \lambda(p) \leq \Lambda(p) \leq \Lambda(\infty)$, we have the desired result.

Lemma. Let $g(x)$ be a differentiable function for $x>0$ and let $g^{\prime}(x)$ be a non-decreasing function (or a non-increasing function) there. If, for some $\alpha<0, L=\lim _{x \rightarrow 0+} g(x) / x^{\alpha}$ exists, then $\lim _{x \rightarrow 0+} g^{\prime}(x) / \alpha x^{\alpha-1}$ also exists and equals $L$.

Proof. Fix $\theta>0$. Then $g(x+\theta x)-g(x) \geq \theta x g^{\prime}(x)$. But

$$
\lim _{x \rightarrow 0+} x^{-\alpha}\{g(x+\theta x)-g(x)\}=L\left\{(1+\theta)^{\alpha}-1\right\} .
$$

Hence $\lim \sup _{x \rightarrow 0+} g^{\prime}(x) / x^{\alpha-1} \leq L \theta^{-1}\left\{(1+\theta)^{\alpha}-1\right\}$ and we may now let $\theta \rightarrow 0$ to get $\lim \sup _{x \rightarrow 0+} g^{\prime}(x) / x^{\alpha-1} \leq \alpha L$. A similar argument shows that $\lim \inf _{x \rightarrow 0+} g^{\prime}(x) / x^{\alpha-1} \geq \alpha L$ and we are done.

ExAmple. Let $f(t)=\sin \log t$. Now

$$
\begin{aligned}
\Gamma(p+1) \Lambda(p)= & \limsup _{x \rightarrow 0+} \int_{0}^{\infty} t^{p} e^{-t} \sin (\log (t / x)) d t \\
= & \limsup _{x \rightarrow 0+}\left\{(\cos \log x) \int_{0}^{\infty} t^{p} e^{-t} \sin \log t d t\right. \\
& \left.\quad-(\sin \log x) \int_{0}^{\infty} t^{p} e^{-t} \cos \log t d t\right\} \\
= & \left|\int_{0}^{\infty} t^{p} e^{-t} \exp (i \log t) d t\right|=|\Gamma(p+1+i)| .
\end{aligned}
$$

Hence

$$
\Lambda(p)=\left|\frac{\Gamma(p+1+i)}{\Gamma(p+1)}\right|
$$

and similary

$$
\lambda(p)=-\left|\frac{\Gamma(p+1+i)}{\Gamma(p+1)}\right| .
$$


In particular, since, as $p \rightarrow-1, \Gamma(p+1) \rightarrow \infty$ and $\Gamma(p+1+i) \rightarrow \Gamma(i)$, we have $\lambda(-1)=\Lambda(-1)=0$. On the other hand, $\Lambda(\infty)=1$, and $\lambda(\infty)=-1$, by Stirling's formula.

\section{The main theorem.}

THEOREM 3.1. $\mathscr{L}(1)=\Lambda(\infty)$ and $l(1)=\lambda(\infty)$.

The following Tauberian theorem of Littlewood is an immediate corollary of this result.

CoRollary. The bounded Lebesgue-measurable function $f$ is Abelaverageable, if and only if it is Cèsaro-averageable, and then av $(f: C)=$ $a v(f: A)$.

Proof. We must prove that $l(0)=\mathscr{L}(0)$ if and only if $\lambda(0)=\Lambda(0)$. Suppose $\lambda(0)=\Lambda(0)$. Then $\lambda(\infty)=\Lambda(\infty)$ (Theorem 2.5). But $\lambda(\infty)=l(1)$ and $\Lambda(\infty)=\mathscr{L}(1)$ (Theorem 3.1) so $l(1)=\mathscr{L}(1)$, and hence $l(0)=\mathscr{L}(0)$ (Theorem 1.5). This is the proof of the hard part of the result. The other part follows directly from the inequalities

$$
l(0) \leq \lambda(0) \leq \Lambda(0) \leq \mathscr{L}(0)
$$

that are derived by a familiar integration by parts in the Laplace transform of $f ; x \int_{0}^{\infty} f(t) e^{-x t} d t=x^{2} \int_{0}^{\infty}\left\{t^{-1} \int_{0}^{t} f(x) d y\right\} t e^{-x t} d t$.

Proof of Theorem 3.1. We prove here that $\mathscr{L}(1)=\Lambda(\infty)$, and first that $\mathscr{L}(1) \geq \Lambda(\infty)$. An easy roundabout proof would be by way of Theorem 4.1 in which we construct a bounded measurable function $f^{*}(x), f^{*}(x) \geq f(x)$, and $a v\left(f^{*}: C\right)=\mathscr{L}(1)$. But the same sort of integration by parts that yields inequalities (3.1) tells us that $\Lambda\left(\infty: f^{*}\right)=$ $\mathscr{L}(1)$. Since $f^{*}(x) \geq f(x), \Lambda\left(\infty: f^{*}\right) \geq \Lambda(\infty)$, and thus $\mathscr{L}(1) \geq \Lambda(\infty)$.

It is important to give a direct proof, because, in certain generalizations of this theory, the analogue of Theorem 4.1 may be false, but the analogue of the present theorem is always true.

To prove directly that $\mathscr{L}(1) \geq \Lambda(\infty)$ it is enough to show that $\mathscr{L}(1) \geq \Lambda(p)$ for each finite positive $p$. We make normalization $0 \leqq f_{(x)} \leqq 1$. Keeping $p$ fixed, for any $\varepsilon>0$ we choose $R$ so large that if $0<A<R^{-1}$, then for all $x$

$$
\frac{F^{(p)}(x)}{I^{(p)}(x)}=\frac{1}{\Gamma(p+1)} \int_{0}^{\infty} t^{p} e^{-t} f(t / x) d t \leq \varepsilon+\frac{1}{\Gamma(p+1)} \int_{A}^{R} t^{p} e^{-t} f(t / x) d t .
$$

Now, for $0<\xi<1$, we put $\sigma_{n}=R \xi^{n}, n=1,2, \cdots$, and choose $N=$ $N(R, \xi)$ so that $\sigma_{N}<R^{-1}$. Let 


$$
M_{n}=\max t^{p} e^{-t} \text { for } \sigma_{n+1} \leq t \leq \sigma_{n} .
$$

We then have

$$
\int_{\sigma_{N}}^{R} t^{p} e^{-t} f(t / x) d t=\sum_{n=0}^{N-1} \int_{\sigma_{n+1}}^{\alpha_{n}} t^{p} e^{-t} f(t / x) d t \leq \sum_{n=0}^{N-1} M_{n} \int_{\sigma_{n+1}}^{\sigma_{n}} f(t / x) d t
$$

We may then conclude that

$$
\limsup _{x \rightarrow 0+} \int_{\sigma_{N}}^{R} t^{p} e^{-t} f(t / x) d t \leq \mathscr{L}(\xi) \sum_{n=0}^{N-1} M_{n}\left(\sigma_{n}-\sigma_{n+1}\right) .
$$

Since $\mathscr{L}(\xi) \leq \mathscr{L}(1)$ we may write

$$
\Lambda(p) \leq \varepsilon+\mathscr{L}(1) \frac{1}{\Gamma(p+1)} \sum_{n=0}^{\infty} M_{n}\left(\sigma_{n}-\sigma_{n+1}\right) .
$$

We now let $\xi \rightarrow 1-$ so that $\sum M_{n}\left(\sigma_{n}-\sigma_{n+1}\right) \rightarrow \int_{0}^{R} t^{p} e^{-t} d t$, then let $R \rightarrow \infty$ and then $\varepsilon \rightarrow 0$ to get $\Lambda(p) \leq \mathscr{L}(1)$.

We now prove that $\Lambda(\infty) \geq \mathscr{L}(1)$. Our proof depends on the fact that when $p$ is large, the function $t^{p} e^{-t}$ has a single and very sharp maximum. We fix $R>0$, then a positive integer $N$, and consider, for $p>R^{2}$, the dissection of the interval $p-R p^{1 / 2} \leq t \leq p+R p^{1 / 2}$ into the $2 N$ subintervals of equal length, $\lambda_{k} \leq t \leq \lambda_{k+1}$, where $-N \leq k<N$. For convenience of notation, we put $\mu_{k}=\lambda_{k+1}$. We see that $\lambda_{k}=$ $p+k \delta p^{1 / 2}$ where $\delta=R / N$. Finally, we put $\xi=\lambda_{-N} / \lambda_{N}$, and $\xi_{k}=\lambda_{k} / \mu_{k}$.

We choose $0<\gamma<1$ and then (for reasons that appear in the proof of Lemma 3.1) choose $\tau$ so that $\gamma<\tau<1$ and $\gamma<\tau^{-1}-2 N\left(\tau^{-1}-\tau^{2}\right)$. We again make the convenient normalization $0 \leq f(x) \leq 1$.

Since, for fixed $R$ and $N$ and each $k, \lim _{p \rightarrow \infty} \xi_{k}=\lim _{p \rightarrow \infty} \xi=1$, we choose $p_{0}$ so that for $p>p_{0}$ we have $\mathscr{L}\left(\xi_{k}\right)>\tau \mathscr{L}(1)$ and $\mathscr{L}(\xi)>\tau \mathscr{L}(1)$.

We now choose a sequence of $x$ tending to $\infty$ for which

$$
\frac{1}{\lambda_{N} x-\lambda_{-N} x} \int_{\lambda_{-N} x}^{\lambda_{N} x} f(t) d t \geq \tau \mathscr{L}(\xi)>\tau^{2} \mathscr{L}(1)
$$

and for which

$$
\frac{1}{\mu_{k} x-\lambda_{k} x} \int_{\lambda_{k} x}^{\mu_{k} x} f(t) d t \leqq \frac{1}{\tau} \mathscr{L}(\xi) \leq \frac{1}{\tau} \mathscr{L}(1) .
$$

Lemma 3.1. For $x$ in the sequence described above

$$
\frac{1}{\mu_{k} x-\lambda_{k} x} \int_{\lambda_{k} x}^{\mu_{k} x} f(t) d t \geq \gamma \mathscr{L}(1) \text {. }
$$

Proof.

$$
\int_{\lambda_{k} x}^{\mu_{k} x} f(t) d t=\int_{\lambda_{-N} x}^{\lambda_{N} x} f(t) d t-\sum_{m \neq k} \int_{\lambda_{m} x}^{\mu_{m} x} f(t) d t
$$


Applying 3.2 and 3.3 , we have

$$
\begin{aligned}
\int_{y_{k} x}^{\mu_{k} x} f(t) d t & \geq \tau^{2}\left(\lambda_{N} x-\lambda_{-N} x\right) \mathscr{L}(1)-\sum_{m \neq k} \frac{\mathscr{L}(1)}{\tau}\left(\mu_{m} x-\lambda_{m} x\right) \\
& =\left(\mu_{k} x-\lambda_{k} x\right) \mathscr{L}(1)\left\{\frac{1}{\tau}-2 N\left(\frac{1}{\tau}-\tau^{2}\right)\right\},
\end{aligned}
$$

since

$$
\mu_{k}-\lambda_{k}=\mu_{m}-\lambda_{m}=\frac{1}{2 N}\left(\lambda_{N}-\lambda_{-N}\right) .
$$

But $\tau$ was chosen to make the expression in brackets exceed $\gamma$, and the proof of the lemma is complete.

Continuing with our proof of the theorem, we put $y=1 / x$ for $x$ in the sequence described above, so that $y \rightarrow 0+$ through some set of values. Now

$$
\int_{0}^{\infty} t^{p} e^{-t y} f(t) d t \geq \int_{\lambda_{-N} x}^{\lambda_{N} x} t^{p} e^{-t y} f(t) d t=\sum_{k=-N}^{-1} \int_{\lambda_{k} x}^{\mu_{k} x}+\sum_{k=0}^{N-1} \int_{\lambda_{k} x}^{\mu_{k} x}=\sum_{1}+\sum_{2} .
$$

In each of the integrals in $\Sigma_{1}$, the variable $t$ of integration lies in the range where $t^{p} e^{-t y}$ is increasing, so that for $-N \leq k<0$

$$
\int_{\lambda_{k} x}^{\mu_{k} x} t^{p} e^{-t y} f(t) d t \geq\left(\lambda_{k} x\right)^{p} e^{-\lambda_{k}} \int_{\lambda_{k} x}^{\mu_{k} x} f(t) d t,
$$

and applying Lemma 3.1, we have

$$
\int_{\lambda_{k} x}^{\mu_{k} x} t^{p} e^{-t y} f(t) d t \geq x^{p+1} \gamma \mathscr{L}(1) \lambda_{k}^{p} e^{-\lambda_{k}}\left(\mu_{k}-\lambda_{k}\right) .
$$

Similarly, for $0 \leq k<N$, we get

$$
\int_{\lambda_{k} x}^{\mu_{k} x} t^{p} e^{-t y} f(t) d t \geq x^{p+1} \gamma \mathscr{L}(1) \mu_{k}^{p} e^{-\mu_{k}}\left(\mu_{k}-\lambda_{k}\right) .
$$

Thus, for $p>p_{0}$,

$$
\Lambda(p) \geq \frac{\gamma \mathscr{L}(1)}{\Gamma(p+1)}\left\{\sum_{k=-N}^{-1} \lambda_{k}^{p} e^{-\lambda_{k}}\left(\mu_{k}-\lambda_{k}\right)+\sum_{k=0}^{N-1} \mu_{k}^{p} e^{-\mu_{k}}\left(\mu_{k}-\mu_{k}\right)\right\} .
$$

We apply Stirling's formula, taking $p_{0}$ also so large that for $p>p_{0}$. $I^{\prime}(p+1) \leq \gamma^{-1} p^{p} e^{-p}(2 \pi p)^{1 / 2}$. Hence

$$
\begin{aligned}
\Lambda(p) & \geq \gamma^{2} \mathscr{L}(1) \frac{\delta}{\sqrt{2 \pi}}\left\{\sum_{k=-N}^{-1}\left(1+\frac{k \delta}{\sqrt{p}}\right)^{p} \exp (-k \delta \sqrt{p})\right. \\
& \left.+\sum_{k=0}^{N-1}\left(1+\frac{(k+1) \delta}{\sqrt{p}}\right)^{p} \exp (-(k+1) \delta \sqrt{p})\right\} .
\end{aligned}
$$

Since 


$$
\lim _{p \rightarrow \infty}\left(1+\frac{k \delta}{\sqrt{p}}\right)^{p} \exp (-k \delta \sqrt{p})=\exp \left(-\frac{(k \delta)^{2}}{2}\right)
$$

we let $p \rightarrow \infty$ above to get

$$
\begin{aligned}
\Lambda(\infty) & \geq \gamma^{2} \mathscr{L}(1) \frac{1}{\sqrt{2 \pi}}\left\{\sum_{k=-N}^{-1} \delta \exp \left(-\frac{(k \delta)^{2}}{2}\right)\right. \\
& \left.+\sum_{k=0}^{N-1} \delta \exp \left(-\frac{((k+1) \delta)^{2}}{2}\right)\right\} .
\end{aligned}
$$

But the expression in brackets is simply an approximating Riemann sum for $\int_{-R}^{R} \exp \left(-x^{2} / 2\right) d x$. Letting first $N \rightarrow \infty$ and then $R \rightarrow \infty$, we have

$$
\Lambda(\infty) \geq \gamma^{2} \mathscr{L}(1) \frac{1}{\sqrt{2 \pi}} \int_{-\infty}^{\infty} \exp \left(-x^{2} / 2\right) d x=\gamma^{2} \mathscr{L}(1) .
$$

Since $\gamma<1$ was arbitrary, we let $\gamma \rightarrow 1$ to complete our proof.

4. The Cèsaro outer and inner means. Let $C$ be the class of Cèsaro-averageable functions, i.e. those for which $\mathscr{L}(0)=l(0)$, and denote the common value by $a v(f: C)$. Let $C^{*}(f)$ be the class of all functions $f^{*} \in C$ for which

$$
f^{*}(x) \geq f(x) \text { for all } x
$$

and

We define

$$
f^{*}(x) \leq \sup _{0<y<\infty} f(y)
$$

$$
\mathscr{L}^{*}=\inf a v\left(f^{*}: C\right), f^{*} \in C^{*}(f)
$$

and call $\mathscr{L}^{*}$ the "Cèsaro outer mean" of $f$. The inner mean $l_{*}$ is similarly defined.

THEOREM 4.1. $\mathscr{L}^{*}=\mathscr{L}(1)$ and $l_{*}=l(1)$. Moreover, there exists an $f^{*} \in C^{*}(f)$ with $a v\left(f^{*}: C\right)=\mathscr{L}(1)$ and there exists an $f_{*} \in C_{*}(f)$ with $\operatorname{av}\left(f_{*}: C\right)=l(1)$.

Proof. That $\mathscr{L}^{*} \geq \mathscr{L}(1)$ is obvious, since, if $f^{*}(x) \geq f(x)$ then $\mathscr{L}\left(1: f^{*}\right) \geq \mathscr{L}(1), \mathscr{L}\left(1: f^{*}\right)=a v\left(f^{*}: C\right)$ by Theorem 1.5, and therefore $a v\left(f^{*}: C\right) \geq \mathscr{L}(1)$. We must now construct our minimizing $f^{*} \in C^{*}(f)$.

Let $\left\{\varepsilon_{k}\right\}$ be a sequence tending to 0 , and $\left\{\lambda_{k}\right\}$ a sequence decreasing to 1 . For each $\lambda_{k}$ there is an $x_{k}>k$ such that

$$
\frac{1}{\lambda_{k} x-x} \int_{x}^{\lambda_{k} x} f(t) d t \leq \mathscr{L}\left(\frac{1}{\lambda_{k}}\right)+\varepsilon_{k} \leq \mathscr{L}(1)+\varepsilon_{k}
$$


for all $x \geq x_{k}$. We shall define a sequence $S_{1}, S_{2}, S_{3}, \cdots$ of finite geometric progressions, and denote by $S=s_{1}, s_{2}, s_{3}, \cdots$ the sequence of numbers we get when we write first all the terms of $S_{1}$, then those of $S_{2}$, those of $S_{3}$, and so on.

Let $S_{1}=x_{1} \lambda_{1}, x_{1} \lambda_{1}^{2}, \cdots, x_{1} \lambda_{1}^{n_{1}}$, where, if we write the last term as $M_{2}=x_{1} \lambda_{1}^{n_{1}}$, we choose $n_{1}$ large enough to make $M_{2}>x_{2}$. We also write $M_{1}=x_{1}$. In general, $S_{k}=M_{k} \lambda_{k}, M_{k} \lambda_{k}^{2}, \cdots, M_{k} \lambda_{k}^{n}$, where $M_{k+1}=M_{k} \lambda_{k}^{n}$, and $n_{k}$ is chosen to make $M_{k+1}>x_{k+1}$.

The important properties of $S$ are

$$
\begin{aligned}
& s_{n} \rightarrow \infty \\
& \frac{s_{n+1}}{s_{n}} \longrightarrow 1 \\
& \frac{1}{s_{n+1}-s_{n}} \int_{s_{n}}^{s_{n+1}} f(t) d t<\mathscr{L}(1)+\delta_{n},
\end{aligned}
$$

as $n \rightarrow \infty$. Here $\left\{\delta_{n}\right\}$ is the sequence whose first $n_{1}$ terms are $\varepsilon_{1}$, whose next $n_{2}$ terms are $\varepsilon_{2}$, and so on, so that $\delta_{n} \rightarrow 0$.

We now define $f^{*}(x)$. For $0<x<\lambda_{1} x_{1}$ put $f^{*}(x)=f(x)$ and then define $f^{*}(x)$ in each of the intervals $s_{n} \leq x<s_{n+1}$ by

$$
f^{*}(x)=\left\{\begin{array}{l}
\sup _{0<y<\infty} f(y) \text { for } s_{n} \leq x<s_{n}+\mu_{n} \\
f(x) \text { for } s_{n}+\mu_{n} \leq x<s_{n+1},
\end{array}\right.
$$

where we choose $\mu=\mu_{n}$ in the interval $0 \leq \mu<s_{n+1}-s_{n}$ so that

$$
\mathscr{L}(1)-\delta_{n} \leq h(\mu) \leq \mathscr{L}(1)+\delta_{n},
$$

where

$$
h(\mu)=\frac{1}{s_{n+1}-s_{n}} \int_{s_{n}}^{s_{n+1}} f^{*}(t) d t .
$$

To see that such a choice of $\mu$ is possible, we first note that if $h(0) \geq \mathscr{L}(1)-\delta_{n}$ then we may choose $\mu=0$, since by $(4.3), h(0) \leq$ $\mathscr{L}(1)+\delta_{n}$. But if $h(0)<\mathscr{L}(1)-\delta_{n}$, we observe that $h(\mu)$ is a continuous function of $\mu$ with

$$
h\left(s_{n+1}-s_{n}\right)=\sup _{0<y<\infty} f(y) \geq \mathscr{L}(1)>\mathscr{L}(1)-\delta_{n}
$$

and we may therefore choose $\mu$ to make $h(\mu)=\mathscr{L}(1)-\delta_{n}$.

Our construction of $f^{*}$ is now complete and it remains only to show that $\lim x^{-1} \int_{0}^{x} f^{*}(t) d t=\mathscr{L}(1)$. But it is easily verified that because of (4.1) and (4.2) we need only show that

$$
\frac{1}{s_{n}} \int_{s_{1}}^{s_{n}} f^{*}(t) d t \longrightarrow \mathscr{L}(1) \text {. }
$$


Now

$$
\begin{aligned}
\frac{1}{s_{n}} \int_{s_{1}}^{s_{n}} f^{*}(t) d t & =\frac{1}{s_{n}} \sum_{k=1}^{n-1} \int_{s_{k}}^{s_{k+1}} f^{*}(t) d t \\
& \leq \frac{1}{s_{n}} \sum_{k=1}^{n-1}\left(\mathscr{L}(1)+\delta_{k}\right)\left(s_{k+1}-s_{k}\right) \\
& =\mathscr{L}(1)\left(1-\frac{s_{1}}{s_{n}}\right)+\frac{1}{s_{n}} \sum_{k=1}^{n-1} \delta_{k}\left(s_{k+1}-s_{k}\right),
\end{aligned}
$$

with a similar inequality in the opposite direction. Now $s_{1} / s_{n} \rightarrow 0$, and an easy estimate shows that

$$
\frac{1}{s_{n}} \sum_{k=1}^{n-1} \delta_{k}\left(s_{k+1}-s_{k}\right) \longrightarrow 0 \text {. }
$$

Hence $a v\left(f^{*}: C\right)=\mathscr{L}(1)$ and we are done.

REMARK. We could similarly define $\Lambda^{*}$, the outer Abel mean, and $\lambda_{*}$, the inner Abel mean, and prove the analogue of Theorem 4.1, namely that $\Lambda^{*}=\Lambda(\infty)$ and $\lambda_{*}=\lambda(\infty)$. The proof would use Theorem 4.1, Theorem 3.1, and its corollary. It would be interesting to find a direct proof that $\Lambda^{*}=\Lambda(\infty)$ without either using these results or essentially reproving them.

\section{BIBLIOGRAPHY}

1. G. H. Hardy, Divergent Series, Oxford, (1949), 170-172.

2. J. E. Littlewood, The converse of Abel's theorem on power series, Proc. London Math. Soc., (2), 9 (1910), 434-48.

3. G. Pólya, Unterschungen über Lücken und Singularitäten von Potenzreihen, Mathematische Zeitschrift, 29 (1929), 549-640.

The Institute For Advanced Study

PRINCETON, NEW JERSEY

THE UNIVERSITY OF ILLINOIS

URBANA, ILLINOIS 



\section{PACIFIC JOURNAL OF MATHEMATICS}

\section{EDITORS}

\author{
David Gilbarg \\ Stanford University \\ Stanford, California \\ F. H. Brownell \\ University of Washington \\ Seattle 5 , Washington
}

\author{
A. L. Whiteman \\ University of Southern California \\ Los Angeles 7, California \\ L. J. PAIGE \\ University of California \\ Los Angeles 24, California
}

\section{ASSOCIATE EDITORS}

\author{
E. F. BECKENBACH \\ T. M. CHERRY \\ D. DERRY
}
E. HEWITT
A. HORN
L. NACHBIN
M. OHTSUKA
H. L. ROYDEN
M. M. SCHIFFER

E. SPANIER

E. G. STRAUS

F. WOLF

\section{SUPPORTING INSTITUTIONS}

\author{
UNIVERSITY OF BRITISH COLUMBIA \\ CALIFORNIA INSTITUTE OF TECHNOLOGY \\ UNIVERSITY OF CALIFORNIA \\ MONTANA STATE UNIVERSITY \\ UNIVERSITY OF NEVADA \\ NEW MEXICO STATE UNIVERSITY \\ OREGON STATE COLLEGE \\ UNIVERSITY OF OREGON \\ OSAKA UNIVERSITY \\ UNIVERSITY OF SOUTHERN CALIFORNIA
}

\author{
STANFORD UNIVERSITY \\ UNIVERSITY OF TOKYO \\ UNIVERSITY OF UTAH \\ WASHINGTON STATE COLLEGE \\ UNIVERSITY OF WASHINGTON

AMERICAN MATHEMATICAL SOCIETY
CALIFORNIA RESEARCH CORPORATION
HUGHES AIRCRAFT COMPANY
SPACE TECHNOLOGY LABORATORIES
NAVAL ORDNANCE TEST STATION

Mathematical papers intended for publication in the Pacific Journal of Mathematics should be typewritten (double spaced), and the author should keep a complete copy. Manuscripts may be sent to any one of the four editors. All other communications to the editors should be addressed to the managing editor, L. J. Paige at the University of California, Los Angeles 24, California. ..

50 reprints per author of each article are furnished free of charge; additional copies may be obtained at cost in multiples of 50 .

The Pacific Journal of Mathematics is published quarterly, in March, June, September, and December. The price per volume (4 numbers) is $\$ 12.00$; single issues, $\$ 3.50$. Back numbers are available. Special price to individual faculty members of supporting institutions and to individual members of the American Mathematical Society: $\$ 4.00$ per volume; single issues, $\$ 1.25$.

Subscriptions, orders for back numbers, and changes of address should be sent to Pacific Journal of Mathematics, 2120 Oxford Street, Berkeley 4, California.

Printed at Kokusai Bunken Insatsusha (International Academic Printing Co., Ltd.), No. $6_{4}$ 2-chome, Fujimi-cho, Chiyoda-ku, Tokyo, Japan.

PUBLISHED BY PACIFIC JOURNAL OF MATHEMATICS, A NON-PROFIT CORPORATION

The Supporting Institutions listed above contribute to the cost of publication of this Journăl, but they are not owners or publishers and have no responsibility for its content or policies. 


\section{Pacific Journal of Mathematics}

\section{Vol. 10, No. $3 \quad$ November, 1960}

Glen Earl Baxter, An analytic problem whose solution follows from a simple

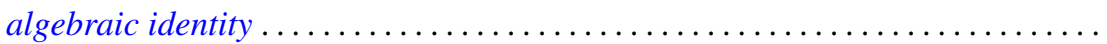

Leonard D. Berkovitz and Melvin Dresher, A multimove infinite game with linear payoff. .

Earl Robert Berkson, Sequel to a paper of A. E. Taylor ......................

Gerald Berman and Robert Jerome Silverman, Embedding of algebraic systems.... 767

Peter Crawley, Lattices whose congruences form a boolean algebra . . . . . ...... 777

Robert E. Edwards, Integral bases in inductive limit spaces . . . . . . . . . . . . . . .

Daniel T. Finkbeiner, II, Irreducible congruence relations on lattices . . . . . . . . . .

William James Firey, Isoperimetric ratios of Reuleaux polygons . . . . . . . . . . . 787

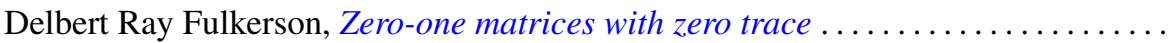

Leon W. Green, A sphere characterization related to Blaschke's conjecture........

Israel (Yitzchak) Nathan Herstein and Erwin Kleinfeld, Lie mappings in

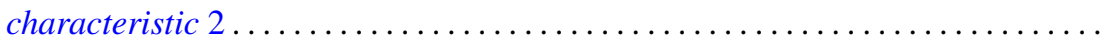

Charles Ray Hobby, A characteristic subgroup of a $p$-group .................

R. K. Juberg, On the Dirichlet problem for certain higher order parabolic

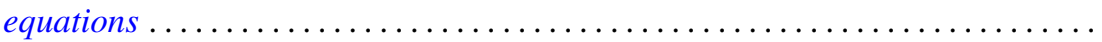

Melvin Katz, Infinitely repeatable games ......................

Emma Lehmer, On Jacobi functions . . . . . . . . . . . . . . . . . . . . . . . . .

D. H. Lehmer, Power character matrices

Henry B. Mann, A refinement of the fundamental theorem on the density of the sum of two sets of integers.

Marvin David Marcus and Roy Westwick, Linear maps on skew symmetric matrices: the invariance of elementary symmetric functions . .

Richard Dean Mayer and Richard Scott Pierce, Boolean algebras with ordered

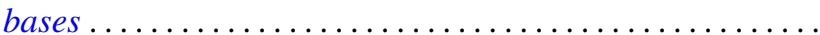

Trevor James McMinn, On the line segments of a convex surface in $E_{3} \ldots$

Frank Albert Raymond, The end point compactification of manifolds ..

Edgar Reich and S. E. Warschawski, On canonical conformal maps of regions of arbitrary connectivity

Marvin Rosenblum, The absolute continuity of Toeplitz's matrices...

Lee Albert Rubel, Maximal means and Tauberian theorems . .

Helmut Heinrich Schaefer, Some spectral properties of positive linear operators

Jeremiah Milton Stark, Minimum problems in the theory of pseudo-conformal transformations and their application to estimation of the curvature of the invariant metric.

Robert Steinberg, The simplicity of certain groups ...

Hisahiro Tamano, On paracompactness. .

Angus E. Taylor, Mittag-Leffler expansions and spectral theory .

Marion Franklin Tinsley, Permanents of cyclic matrices ...... . 